\title{
Structure-function relationship of inducer peptide pheromones involved in bacteriocin production in Carnobacterium maltaromaticum and Enterococcus faecium
}

\author{
Correspondence \\ John C. Vederas \\ john.vederas@ualberta.ca
}

Received 18 April 2007

Revised 3 July 2007

Accepted 16 July 2007

\author{
Marco J. van Belkum, ${ }^{1}$ Darren J. Derksen, ${ }^{1}$ Charles M. A. P. Franz ${ }^{2}$ \\ and John C. Vederas ${ }^{1}$ \\ ${ }^{1}$ Department of Chemistry, University of Alberta, Edmonton, AB T6G 2G2, Canada
${ }^{2}$ Federal Research Centre for Nutrition and Food, Institute of Hygiene and Toxicology, Haid-und-
Neu-Strasse 9, D-76131 Karlsruhe, Germany
}

The production of several bacteriocins in lactic acid bacteria is regulated by inducer peptide pheromones that specifically interact with their cognate bacterial receptor. These peptide pheromones are between 19 and 27 aa long and contain a conserved (V/I)-X-X-X-F sequence followed by positively charged residues in the C-terminal domain. $\mathrm{CbaX}$ and EntF are peptide pheromones that share similarity and are involved in the production of carnobacteriocin $A$ in Carnobacterium maltaromaticum LV17A and enterocins A and B in Enterococcus faecium CTC492, respectively. CbaX, EntF and two hybrids, CbaX : : EntF and EntF ::CbaX, were tested for pheromone activity in LV17A and CTC492. EntF and EntF : : CbaX only induced bacteriocin production in CTC492, whereas $\mathrm{CbaX}$ and $\mathrm{CbaX}$ : : EntF induced carnobacteriocin A production in LV17A and, at high concentrations, also cross-induced enterocin production in CTC492. Various peptide fragments of $\mathrm{CbaX}$ and EntF were made for further structure-function analysis. The $\mathrm{C}$-terminal fragments, but not the $\mathrm{N}$-terminal fragments, were able to effect bacteriocin induction. The 10-mer EntF(16-25), derived from the C-terminal domain of EntF, showed pheromone activity in LV17A. In contrast, the C-terminal 9-mer of CbaX, CbaX(16-24), inhibited pheromone activity in both LV17A and CTC492. EntF(16-25) and CbaX(16-24) differ by two amino acids. Changing either one of these abolished pheromone activity as well as the ability to inhibit pheromone activity. These results indicate that the C-terminal domain of these peptide pheromones interacts relatively non-specifically with the receptor, and that induction is greatly facilitated by the $\mathrm{N}$-terminal domain that recognizes specifically its cognate receptor.

\section{INTRODUCTION}

Many bacteria are able to communicate with each other in a cell-density-dependent manner, called quorum sensing, by the secretion of signalling molecules. These signalling molecules are detected by other bacteria when they reach a certain concentration and change the expression level of specific genes that enable the cells to adapt in a coordinated manner to their environment (Bassler \& Losick, 2006; Keller \& Surette, 2006; Reading \& Sperandio, 2006). In Gram-positive bacteria, the signalling molecules are often inducer peptide pheromones (Kleerebezem \& Quadri, 2001). The discovery that bacteriocin production in Carnobacterium maltaromaticum LV17 and Lactobacillus plantarum C11 was dependent on cell density indicated that quorum sensing plays a role in the production of class

Abbreviations: APT, All Purpose Tween; CD, circular dichroism; LAB, lactic acid bacteria; TFA, trifluoroacetic acid; TFE, trifluoroethanol.
II bacteriocins in lactic acid bacteria (LAB) (Diep et al., 1995; Saucier et al., 1995). Class II bacteriocins are small, cationic, antibacterial peptides that do not contain unusual amino acids and permeabilize the membranes of sensitive, usually closely related bacteria (Klaenhammer, 1993; Nes et al., 1996; van Belkum \& Stiles, 2000). It has been shown that the production of several, but not all, class II bacteriocins in LAB is regulated by peptide pheromones (van Belkum \& Stiles, 2000). The gene encoding the peptide pheromone is often genetically linked to genes encoding the histidine protein kinase and the response regulator involved in bacteriocin expression (Kleerebezem \& Quadri, 2001). The peptide pheromone is produced as a precursor with a double-glycine-leader peptide that is recognized and cleaved off by the bacteriocin $A B C$ transporter during translocation out of the cell (Håvarstein et al., 1995; van Belkum et al., 1997). These peptide pheromones vary in length between 19 and 27 aa 
and are detected by the histidine protein kinase that is located in the cell membrane. The $\mathrm{N}$-terminal domain of the histidine protein kinase contains six or seven membranespanning segments that act as the sensor of the peptide pheromone (Johnsborg et al., 2003). Interaction of the peptide pheromone with the histidine protein kinase activates the kinase, enabling it to phosphorylate the response regulator. The response regulator in turn activates the bacteriocin operon(s) (Kleerebezem \& Quadri, 2001; Rohde \& Quadri, 2006).

The interaction between receptor and peptide pheromone is highly specific and peptide pheromones only induce the cognate bacteriocin expression system of the receptor (Kleerebezem et al., 1997). In this study we investigated peptide pheromones from Carnobacterium and Enterococcus species that share some homology in their amino acid sequence. C. maltaromaticum LV17A, a derivative of strain LV17, produces the bacteriocin carnobacteriocin A (Ahn \& Stiles, 1992; Worobo et al., 1994) and the production of this bacteriocin is regulated by the 24-mer inducer peptide CbaX (Franz et al., 2000). Bacteriocins enterocin A and B are produced by a great variety of Enterococcus faecium strains (Franz et al., 2007). In several strains the expression of these two bacteriocins is regulated by the peptide pheromone EntF which consists of 25 aa. E. faecium CTC492 produces both enterocins A and B and both are induced by EntF (Nilsen et al., 1998). Here we report that, at higher than normal concentrations of peptide pheromones, cross-induction between these two bacteriocin expression systems is possible and that the Nterminal domain of these peptides plays a role in recognition of its cognate receptor. Furthermore, we show that the C-terminal fragments of these peptide pheromones are able to cross-induce bacteriocin production or crossinterfere with pheromone activity, indicating that the Cterminal domain of these peptides interacts relatively nonspecifically with the receptor molecule.

\section{METHODS}

Induction peptide synthesis, purification and characterization. Peptides were synthesized on pre-loaded resins [H-Cys(Trt)-2-Cl-Trt, Fmoc-Asn(Trt)-Wang or Fmoc-Ser $(O-t \mathrm{Bu})$-Wang; Novabiochem] with resin loading between 0.54 and $0.75 \mathrm{mmol} \mathrm{g}^{-1}$ on a $0.1-$ $0.5 \mathrm{mmol}$ scale. All peptides were synthesized manually using standard Fmoc solid-phase peptide syntheses (SPPS) protocols. Removal of Fmoc was completed using $20 \%$ piperidine/DMF for $5 \mathrm{~min}$ and was repeated until either a Kaiser test (Kaiser et al., 1970) was clearly positive or background absorbance was reached by UV detection at $301 \mathrm{~nm}$. Side chain protecting groups for amino acids or derivatives were as follows: $\mathrm{Asn}(N-\mathrm{Trt}), \mathrm{Glu}(O-t \mathrm{Bu}), \mathrm{G} \ln (N-\mathrm{Trt})$, Lys $(N$-Boc $), \operatorname{Ser}(O-t \mathrm{Bu})$ and $\operatorname{Thr}(O-t \mathrm{Bu})$. Cysteine residues were introduced using Fmoc-Cys(Trt)-OPfp esters. Pseudoproline (Mutter et al., 1995) (oxazolidine) dipeptides [Fmoc-Ala-Thr $\left(\psi^{\mathrm{Me}, \mathrm{Me}}\right.$ pro)-OH, Fmoc-Ser(O- $t \mathrm{Bu})-\operatorname{Ser}\left(\psi^{\mathrm{Me}, \mathrm{Me}}\right.$ pro $)-\mathrm{OH}, \quad$ Fmoc-Phe-Ser $\left(\psi^{\mathrm{Me}, \mathrm{Me}}\right.$ pro $)$ $\mathrm{OH}$; Novabiochem] were incorporated whenever possible, except when this would have resulted in contiguous couplings. Cleavage and deprotection of the peptide from the resin was accomplished by treatment with a freshly prepared solution of TFA/ $\mathrm{H}_{2} \mathrm{O} /$ triisopropyl silane ( $95: 2.5: 2.5$, by vol.) mixture for $4 \mathrm{~h}$ with mechanical stirring. Filtration, followed by concentration in vacuo and trituration with cold diethyl ether afforded the crude peptides as white solids. Peptides were purified using a Delta-Pak C18 Prep-Pak Cartridge (Waters) installed on a Gilson HPLC (Gilson 322 pump, UV-Vis 152 detector; Mandel Scientific). Purification was achieved using acetonitrile and water containing $0.1 \%$ TFA at a flow rate of $10 \mathrm{ml} \mathrm{min}^{-1}$ with UV detection at $220 \mathrm{~nm}$. The acetonitrile gradient either was increased from 20 to $50 \%$ over 25 min followed by a ramp to $90 \%$ acetonitrile over $5 \mathrm{~min}$, or was varied from 5 to $95 \%$ over $46 \mathrm{~min}$. Fractions containing appropriate mass by MALDI-TOF MS were concentrated, lyophilized and repurified to homogeneity. Compounds were purified to single peak by HPLC and MALDI-TOF within $0.3 \mathrm{Da}(\mathrm{M}+\mathrm{H})$. All mass spectral analyses were performed on a Perspective Biosystems Voyager Elite MALDI-TOF mass spectrometer with delayed extraction in reflectron mode. The two-layer method (Dai et al., 1996, 1999) using 3,5-dimethoxy-4-hydroxycinnamic acid (sinapinic acid) as the matrix was used for all MALDI-MS samples.

Bacteriocin activity assay. C. maltaromaticum strains LV17A and LV17C were grown in APT (All Purpose Tween) broth (Difco) at $25{ }^{\circ}$ C. E. faecium CTC492 and Lactobacillus sakei DSM 20017 were grown in APT broth at $30^{\circ} \mathrm{C}$. Bacteriocin activity was determined using heat-treated cell-free supernatant of the producer organism in a spot-on-lawn test (Franz et al., 2000). The critical dilution method was used to measure bacteriocin titre $\left(\mathrm{AU} \mathrm{ml} \mathrm{m}^{-1}\right)$ as described by Franz et al. (1996). C. maltaromaticum LV17C (Ahn \& Stiles, 1992), a derivative of LV17A cured of its bacteriocin plasmid, was used as a sensitive indicator for carnobacteriocin A. L. sakei DSM 20017 was used as an indicator organism against enterocins A and $\mathrm{B}$.

Peptide pheromone activity. To lose bacteriocin production, fully grown cultures of C. maltaromaticum LV17A and E. faecium CTC492 were diluted $10^{6}$-fold in $5 \mathrm{ml} \mathrm{APT}$ and allowed to grow until turbid. Peptides to be tested for pheromone activity (see Table 1) were serial diluted in concentrations ranging from $10^{-5}$ to $10^{-13} \mathrm{M}$ in $2.5 \mathrm{ml}$ APT. A $1 \%$ inoculum of the bacteriocin non-producing culture LV17A or CTC492 was added to the broth containing the peptides and allowed to grow for $24 \mathrm{~h}$. As a negative control, no peptides were added to bacteriocin non-producing cultures of LV17A and CTC492. The supernatants of these cultures were tested for bacteriocin activity by the spot-on-lawn assay using LV17C or DSM 20017 as indicators.

Peptide fragments of $\mathrm{CbaX}$ and EntF to be tested for inhibition of pheromone activity of $\mathrm{CbaX}$ and EntF were added to $2.5 \mathrm{ml}$ APT in concentrations ranging from $10^{-5}$ to $10^{-7} \mathrm{M}$. The peptide-containing APT broth was subsequently inoculated with $1 \%$ of a bacteriocin non-producing culture of LV17A or CTC492 and supplemented with $5 \times 10^{-11} \mathrm{M} \mathrm{CbaX}$ or EntF, respectively. The bacteriocin titre of these cultures was measured after $24 \mathrm{~h}$ growth as described above using LV17C or DSM 20017 as indicators.

Circular dichroism (CD) spectroscopy. CD spectra were recorded using an Olis DSM 17 instrument. All measurements were obtained using a peptide concentration of $5 \times 10^{-5} \mathrm{M}$ in $10 \mathrm{mM}$ potassium phosphate ( $\mathrm{pH} 7.4)$ using various concentrations of trifluoroethanol $(0-80 \%, v / v)$. Measurements were obtained at $23{ }^{\circ} \mathrm{C}$ using a quartz microcell cuvette (Hellma) with a path length of $0.2 \mathrm{~mm}$. Samples were scanned five times at $20 \mathrm{~nm} \mathrm{~min}{ }^{-1}$ from 190 to $260 \mathrm{~nm}$. The scans were averaged and corrected with respect to the baseline. The $\alpha$ helical content of the peptide was estimated based on the value of the molar ellipticity $(\theta)$ at $222 \mathrm{~nm}$ using a modified equation from Morrow et al. (2000):

Percentage $\alpha$-helix $=\left[\left(-[\theta]_{222 \mathrm{~nm}}+3000\right) / 39000\right] \times 100$ 
Table 1. Amino acid sequences of various peptides and their ability to induce bacteriocin production in C. maltaromaticum LV17A and E. faecium CTC492

\begin{tabular}{|c|c|c|c|}
\hline \multirow[t]{2}{*}{ Peptide } & \multirow[t]{2}{*}{ Sequence $^{*}$} & \multicolumn{2}{|c|}{$\begin{array}{l}\text { Concentration of peptide }(\mathrm{M}) \\
\text { required for bacteriocin induction of }\end{array}$} \\
\hline & & LV17A & CTC492 \\
\hline CbaX & SINSQIGKATSSISKCVFSFFKKC & $10^{-11}$ & $10^{-7}$ \\
\hline EntF & AGTKPQGKPASNLVECVFSLFKKCN & - & $10^{-11}$ \\
\hline CbaX:: EntF & SINSQIGKATSNLVECVFSLFKKCN & $10^{-9}$ & $10^{-7}$ \\
\hline EntF : : CbaX & AGTKPQGKPASSISKCVFSFFKKC & - & $10^{-11}$ \\
\hline $\operatorname{CbaX}(7-24)$ & GKATSSISKCVFSFFKKC & $10^{-6}$ & - \\
\hline $\operatorname{CbaX}(11-24)$ & SSISKCVFSFFKKC & - & - \\
\hline $\operatorname{CbaX}(16-24)$ & CVFSFFKKC & - & - \\
\hline $\operatorname{CbaX}(16-24+\mathrm{N})$ & CVFSFFKKCN & - & - \\
\hline $\operatorname{CbaX}(1-11)$ & SINSQIGKATS & - & - \\
\hline $\operatorname{EntF}(7-25)$ & GKPASNLVECVFSLFKKCN & $10^{-5}$ & $10^{-6}$ \\
\hline $\operatorname{EntF}(11-25)$ & SNLVECVFSLFKKCN & $10^{-5}$ & - \\
\hline $\operatorname{EntF}(16-25)$ & CVFSLFKKCN & $10^{-6}$ & - \\
\hline $\operatorname{EntF}(16-25-N)$ & CVFSLFKKC & - & - \\
\hline $\operatorname{EntF}(1-11)$ & AGTKPQGKPAS & - & - \\
\hline
\end{tabular}

${ }^{\star}$ EntF amino acid sequences are shown in bold type.

\section{RESULTS}

\section{Pheromone activity of CbaX and EntF and their derivatives}

The amino acid sequences of a number of inducer peptide pheromones responsible for the production of class II

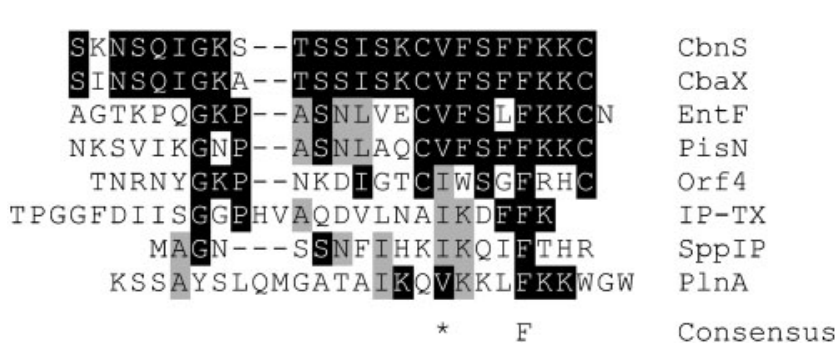

Fig. 1. Amino acid sequence alignment of inducer peptide pheromones involved in bacteriocin production in LAB. Conserved amino acids are shown in shaded blocks. * indicates the position of a conserved $\mathrm{V} / \mathrm{I}$ residue. The peptide pheromones CbnS, CbaX, EntF, PisN, Orf-4, IP-TX, SppIP and PlnA are involved in the production of carnobacteriocin $\mathrm{B} 2$ in C. maltaromaticum LV17B (Kleerebezem et al., 2001), carnobacteriocin A in C. maltaromaticum LV17A (Franz et al., 2000), enterocins A and $B$ in E. faecium CTC492 (Nilsen et al., 1998), piscicolin 126 in C. maltaromaticum UAL26 (Gursky et al., 2006), sakacin A in L. sakei Lb706 (Axelsson \& Holck, 1995), sakacin TX in L. sakei 5 (Vaughan et al., 2003), sakacin P in L. sakei LTH673 (Eijsink et al., 1996) and plantaricin A in L. plantarum C11 (Diep et al., 1995), respectively. bacteriocins were compared (Fig. 1). Significant homology, especially in the C-terminal domain, can be seen between peptide pheromones produced by C. maltaromaticum and E. faecium. Furthermore, all peptides shown contain a conserved V/I-X-X-X-F sequence near the C-terminal end, followed by positively charged residues. Given the known specificity of peptide pheromones for their cognate receptors and the high homology between pheromones $\mathrm{CbaX}$ and EntF, we decided to investigate whether crossinduction could take place in C. maltaromaticum LV17A and E. faecium CTC492. Peptides CbaX and EntF were synthesized and used to induce bacteriocin production in both LV17A and CTC492 with peptide concentrations as high as $10^{-5} \mathrm{M}$. Both $\mathrm{CbaX}$ and EntF were able to induce their cognate bacteriocin production at $10^{-11} \mathrm{M}$ in LV17A and CTC492, respectively (Table 1). EntF was unable to induce bacteriocin production in LV17A at a concentration of up to $10^{-5} \mathrm{M}$. However, $\mathrm{CbaX}$ induced bacteriocin production in CTC492 at $10^{-7} \mathrm{M}$ (Table 1), indicating that at high concentrations this peptide pheromone is able to cross-induce bacteriocin production in E. faecium CTC492. To determine the role of the $\mathrm{N}$ - and C-terminal domains of these peptides in pheromone activity, two hybrid peptides were made. CbaX::EntF contains the first $10 \mathrm{~N}$-terminal amino acids from $\mathrm{CbaX}$ and the $\mathrm{C}$-terminal 15 amino acids from EntF, whereas EntF:: CbaX contains the first $10 \mathrm{~N}$ terminal amino acids from EntF and the C-terminal 14 amino acids from CbaX (Table 1). Like EntF, EntF : : CbaX had no pheromone activity in LV17A. In contrast, bacteriocin production in LV17A could be induced by CbaX:: EntF at $10^{-9} \mathrm{M}$, indicating that the $\mathrm{N}$-terminal domain is important for recognition by the cognate 
receptor. This was confirmed by induction experiments in CTC492 that showed higher pheromone activity of EntF : : CbaX than CbaX:: EntF (Table 1).

To elucidate the role different parts of the peptide pheromones play in bacteriocin induction, various $\mathrm{N}$ and C-terminal fragments derived from the sequences of $\mathrm{CbaX}$ and EntF were synthesized. Peptides $\mathrm{CbaX}(7-24)$, $\mathrm{CbaX}(11-24)$ and $\mathrm{CbaX}(16-24)$ consisted of the Cterminal 18, 14 and 9 amino acids of CbaX, respectively, whereas $\operatorname{EntF}(7-25), \operatorname{EntF}(11-25)$ and $\operatorname{EntF}(16-25)$ consisted of the C-terminal 19, 15 and 10 amino acids of EntF, respectively. In addition, $\operatorname{CbaX}(1-11)$ and $\operatorname{EntF}(1-11)$ were made based on the $\mathrm{N}$-terminal 11 amino acids of $\mathrm{CbaX}$ and EntF, respectively. As a result, all peptide fragments started or ended with amino acid residues that are identical in CbaX and EntF (see Table 1). These peptides were subsequently screened for pheromone activity in LV17A and CTC492 at concentrations up to $10^{-5} \mathrm{M}$. $\operatorname{CbaX}(7-24)$ and $\operatorname{EntF}(7-25)$ were able to induce their own cognate bacteriocin systems in LV17A and CTC492, respectively, at a concentration of up to $10^{-6} \mathrm{M}$ (Table 1 ). None of the other $\mathrm{CbaX}$ fragments showed any pheromone activity in LV17A or CTC492. However, the C-terminal 19-, 15- and 10-mers of EntF showed pheromone activity in LV17A (Table 1). Neither of the N-terminal 11-mers of EntF and $\mathrm{CbaX}$ was able to induce bacteriocin production (Table 1). $\mathrm{CbaX}(16-24)$ and $\operatorname{EntF}(16-25)$ differ by only two amino acids as $\operatorname{EntF}(16-25)$ has an additional asparagine residue at its $\mathrm{C}$ terminus and a leucine residue instead of a phenylalanine. Given the fact that $\operatorname{EntF}(16-25)$ but not CbaX(16-24) induced bacteriocin production in LV17A, two intermediate peptides, $\mathrm{CbaX}(16-24+\mathrm{N})$ and $\operatorname{EntF}(16-$ $25-\mathrm{N}$ ), were synthesized (Table 1). Neither of these two peptides showed pheromone activity in LV17A and CTC492, indicating that these single amino acid changes in $\operatorname{EntF}(16-25)$ abolished pheromone activity.

All peptides described above were tested at a concentration of $10^{-3} \mathrm{M}$ for antimicrobial activity towards LV17C and DSM 20017 using the spot-on-lawn technique. None of the peptides caused inhibition of growth of the two LAB strains (data not shown).

\section{C-terminal peptide fragment of $\mathrm{CbaX}$ inhibits pheromone activity}

Various peptide pheromone fragments were tested for their ability to inhibit pheromone activity by $\mathrm{CbaX}$ and EntF. Both $\mathrm{CbaX}$ and EntF were used at a concentration of $5 \times 10^{-11} \mathrm{M}$ to induce bacteriocin production in LV17A and CTC492, respectively. The concentration of the peptide fragments used in these inhibition studies was again up to $10^{-5} \mathrm{M}$. The two $\mathrm{N}$-terminal fragments $\mathrm{CbaX}(1-11)$ and $\operatorname{EntF}(1-11)$ as well as $\mathrm{CbaX}(11-24)$ and $\operatorname{EntF}(11-25)$ were unable to interfere with bacteriocin induction in LV17A and CTC492, respectively (data not shown). However, the shortest C-terminal fragment of CbaX was able to inhibit pheromone activity in both strains. At $10^{-5} \mathrm{M}, \mathrm{CbaX}(16-24)$ reduced bacteriocin production in LV17A and at up to $10^{-6} \mathrm{M}$ the peptide also reduced or completely inhibited bacteriocin production in CTC492 (Table 2). In contrast, the shortest Cterminal fragment of EntF, $\operatorname{EntF}(16-25)$, was unable to inhibit pheromone activity in CTC492 (data not shown). Neither $\operatorname{CbaX}(16-24+\mathrm{N})$ nor $\operatorname{EntF}(16-25 \mathrm{~N})$ affected pheromone activity in LV17A and CTC492 (data not shown), indicating that at the concentrations used only CbaX(16-24) has the potential to antagonize pheromone activity of CbaX and EntF.

\section{CD spectra analysis}

The full-length peptides were dissolved at a concentration of $5 \times 10^{-5} \mathrm{M}$ in a variety of solvent mixtures of trifluoroethanol (TFE) and $10^{-2} \mathrm{M}$ potassium phosphate buffer ( $\mathrm{pH}$ 7.4), varying the TFE concentration from 0 to $80 \%$. At concentrations of 0 and $20 \%$ TFE, the samples did not assume any conformation consistent with $\alpha$-helical peptide structure (data not shown). At concentrations of 40, 60 and $80 \%$ TFE, the CD spectra indicate that the parent peptides $\mathrm{CbaX}$ and $\mathrm{EntF}$ display relatively constant secondary structure ranging from $32-44 \%$ and $15-18 \% \alpha-$ helical content, respectively (Table 3 ). It is interesting to note that the CD spectra of the hybrid peptides respond much more to TFE concentration. There is a much wider range of $\alpha$-helical content in hybrids: $27-52 \%$ for the CbaX:: EntF hybrid and 9-28\% for EntF : : CbaX (Table 3). In general, the secondary structures of $\mathrm{CbaX}$ and CbaX::EntF show a higher $\alpha$-helical content in a membrane-mimicking environment compared to EntF and EntF::CbaX. These structural data appear to exhibit a correlation to the activity of these peptides, as the $\mathrm{CbaX}$ and CbaX::EntF peptides were both able to induce bacteriocin production in LV17A and cross-induce at similar levels bacteriocin production in CTC492. Similarly, the CD spectra of EntF and EntF : : CbaX correlate well with their pheromone activity profile.

Table 2. Inhibitory effect of $\mathrm{CbaX}(16-24)$ on pheromone activity of CbaX in C. maltaromaticum LV17A and EntF in $E$. faecium CTC492

\begin{tabular}{|c|c|c|}
\hline $\begin{array}{l}\text { Concentration of } \\
\mathrm{CbaX}(16-24)(\mathrm{M})\end{array}$ & 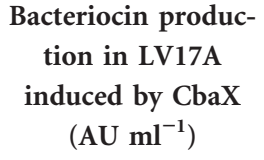 & $\begin{array}{c}\text { Bacteriocin produc- } \\
\text { tion in CTC492 } \\
\text { induced by EntF } \\
\left(\mathrm{AU} \mathrm{m} \mathrm{ml}^{-1}\right)\end{array}$ \\
\hline 0 & 6400 & 800 \\
\hline $10^{-5}$ & 800 & 0 \\
\hline $10^{-6}$ & 6400 & 200 \\
\hline $10^{-7}$ & 6400 & 800 \\
\hline
\end{tabular}


Table 3. $\alpha$-Helical content of natural and hybrid peptide pheromones in varying concentrations of TFE in phosphate buffer ( $\mathrm{pH} 7.4)$

\begin{tabular}{|lccc|}
\hline $\begin{array}{l}\text { Peptide } \\
\left(\mathbf{5} \times \mathbf{1 0}^{-\mathbf{5}} \mathbf{M}\right)\end{array}$ & $\begin{array}{c}\text { TFE : phosphate } \\
\text { buffer }(\mathbf{p H} \text { 7.4) } \\
(\%)\end{array}$ & $\begin{array}{c}\text { Molar ellipticity } \\
\text { at } 222 \mathbf{~ n m}\end{array}$ & $\begin{array}{c}\boldsymbol{\alpha} \text {-Helix } \\
(\%)^{*}\end{array}$ \\
\hline CbaX & 40 & -9320.36 & 32 \\
& 60 & -12033.78 & 39 \\
& 80 & -14014.60 & 44 \\
CbaX : : EntF & 40 & -17421.24 & 52 \\
& 60 & -7700.73 & 27 \\
& 80 & -12476.15 & 40 \\
EntF :: CbaX & 40 & -3444.39 & 17 \\
& 60 & -7974.83 & 28 \\
& 80 & -643.17 & 9 \\
EntF & & & \\
& 40 & -3271.62 & 16 \\
& 60 & -2965.30 & 15 \\
& 80 & -3960.83 & 18 \\
\hline
\end{tabular}

${ }^{\star}$ Calculated as described in Methods.

\section{DISCUSSION}

Inducer peptide pheromones play an essential role in the production of a number of bacteriocins in LAB. These peptide pheromones specifically induce their cognate bacteriocin expression system (Kleerebezem et al., 1997). In our study we were able to cross-induce the expression of enterocins in E. faecium CTC492 with CbaX from $C$. maltaromaticum LV17A. To our knowledge this is the first example of cross-induction between different bacteriocin expression systems using non-hybrid histidine protein kinases. However, it probably cannot be described as crosstalk between species, as the amount of $\mathrm{CbaX}$ required to induce enterocin production in CTC492 is significantly higher (4 logs) than is required with EntF. When spent supernatant of C. maltaromaticum LV17A is used, no bacteriocin induction in CTC492 is observed, indicating that the amount of CbaX produced by LV17A in the supernatant is not enough to activate enterocin production in CTC492 (data not shown).

Experiments with the $\mathrm{N}$ - and C-terminal fragments of CbaX and EntF showed that the C-terminal 18-mer of CbaX and 19-mer of EntF, but not the N-terminal 11-mers of $\mathrm{CbaX}$ and EntF were able to induce their cognate bacteriocin system in LV17A and CTC492. When shorter versions of the C-terminal 18- and 19-mer of CbaX and EntF were used, the peptides lost the ability to induce their cognate bacteriocin systems. These results seem to contradict studies with the peptide pheromone plantaricin A (Kristiansen et al., 2005). Plantaricin A is produced by $L$. plantarum C11 in three variants: as a 26 aa full-length peptide and as N-terminal truncated forms of 23 and 22 aa.
Interestingly, all three variants of plantaricin A also have antimicrobial activity. Induction studies showed that at high concentrations, the N-terminal 5-mer of plantaricin A induced bacteriocin activity that was dependent on the chirality of the peptide, as only the L- but not the Denantiomeric form had pheromone activity (Kristiansen et al., 2005). Furthermore, the L- and D-forms of the Cterminal 17-mer of plantaricin A were able to inhibit pheromone activity by plantaricin A, indicating that the inhibition of induction by these peptides was not dependent on chirality (Kristiansen et al., 2005). Unexpectedly, the C-terminal 19-, 15- and 10-mers of EntF cross-induced bacteriocin production in LV17A. The 10-mer had even more pheromone activity than the 19- or 15-mer peptides as the concentration of $\operatorname{EntF}(16-25)$ needed to induce carnobacteriocin A in LV17A was 10fold lower than that of $\operatorname{EntF}(11-25)$ and $\operatorname{EntF}(7-25)$. This would indicate that the interaction of the C-terminal domain of these peptide pheromones is not necessarily very specific towards its own cognate receptor. This seems to be confirmed by the fact that the C-terminal 9-mer of CbaX, $\mathrm{CbaX}(16-24)$, is able to inhibit pheromone activity of CbaX as well as EntF in LV17A and CTC492, respectively. Apparently, the interaction of $\operatorname{CbaX}(16-24)$ with the receptor does not induce a conformational change that would activate the sensor, but the interaction is enough to compete with the pheromone activity of CbaX and EntF. The fact that these domains can interact with receptors from different bacteriocin systems might explain why chirality does not play a role in recognition of the $\mathrm{C}$ terminal domain by the receptor as was observed for plantaricin A (Kristiansen et al., 2005). However, the results with $\mathrm{CbaX}(16-24+\mathrm{N})$ and $\operatorname{EntF}(16-25-\mathrm{N})$ show that a small change in the amino acid sequence can abolish interaction of the C-terminal domain with the receptor. NMR analysis of plantaricin A revealed that the C-terminal domain has an amphiphilic $\alpha$-helical structure in a lipid environment, suggesting an electrostatic interaction with the membrane surface near the receptor (Kristiansen et al., 2005). The CD spectra of CbaX and CbaX::EntF show considerable $\alpha$-helical content in the presence of structureinducing solvents such as TFE, but this is less apparent for the other peptides synthesized in the current study. However, given the homology in the C-terminal domain of these peptide pheromones with plantaricin $\mathrm{A}$, it is possible that a similar amphiphilic $\alpha$-helical structure may be produced in the C-terminal domain of $\mathrm{CbaX}$ and EntF. The possibility that these peptide pheromones interact with the receptor via the membrane environment is in line with the suggestion that nisin, an amphiphilic lantibiotic bacteriocin produced by Lactococcus lactis, induces its own production by inserting itself in the membrane and activating the histidine protein kinase NisK (Abbas Hilmi et al., 2006).

The induction experiments in CTC492 with the full-length peptide pheromones and their hybrids revealed that the $\mathrm{N}$ terminal domain plays a major role in the recognition of 
the peptide pheromone by its cognate receptor. The results with $\mathrm{CbaX}$ and $\mathrm{CbaX}:$ : EntF showed that replacing the EntF N-terminal domain by the $\mathrm{CbaX} \mathrm{N}$-terminal domain greatly reduced pheromone activity in CTC492. The ability to induce bacteriocin production in LV17A was completely abolished when the CbaX N-terminal domain was replaced by the EntF N-terminal domain in EntF:: CbaX and EntF. These results, and the finding that the chirality of the $\mathrm{N}$ terminal domain of plantaricin A is important for pheromone activity (Kristiansen et al., 2005), indicate that the specific interaction of the $\mathrm{N}$ terminus with the receptor explains why these peptide pheromones only induce their cognate bacteriocin system. Taken together, peptide pheromones seem to use their amphiphilic C-terminal domain to bind to the histidine protein kinase, possibly at membrane level, in a relative non-specific fashion. The weak interaction is greatly improved by the N-terminal domain of the peptide pheromone that is highly specific for the cognate receptor. This interaction will enable the peptide pheromone to activate bacteriocin production at less than nanomolar level.

\section{ACKNOWLEDGEMENTS}

We would like to thank Wayne Moffat for assistance in obtaining CD spectra. Financial support by the Natural Sciences and Engineering Research Council of Canada (NSERC), the Advanced Foods and Biomaterials Network (AFMNet), the Canada Research Chair in Bioorganic and Medicinal Chemistry and the Alberta Ingenuity Fund are gratefully acknowledged.

\section{REFERENCES}

Abbas Hilmi, H. T., Kylä-Nikkilä, K., Ra, R. \& Saris, P. E. J. (2006). Nisin induction without nisin secretion. Microbiology 152, 1489-1496.

Ahn, C. \& Stiles, M. E. (1992). Mobilization and expression of bacteriocin plasmids from Carnobacterium piscicola isolated from meat. J Appl Bacteriol 73, 217-228.

Axelsson, L. \& Holck, A. (1995). The genes involved in production of and immunity to sakacin A, a bacteriocin from Lactobacillus sake Lb706. J Bacteriol 177, 2125-2137.

Bassler, B. L. \& Losick, R. (2006). Bacterially speaking. Cell 125, 237-246.

Dai, Y. Q., Whittal, R. M. \& Li, L. (1996). Confocal fluorescence microscopic imaging for investigating the analyte distribution in MALDI matrices. Anal Chem 68, 2494-2500.

Dai, Y., Whittal, R. M. \& Li, L. (1999). Two-layer sample preparation: a method for MALDI-MS analysis of complex peptide and protein mixtures. Anal Chem 71, 1087-1091.

Diep, D. B., Håvarstein, L. S. \& Nes, I. F. (1995). A bacteriocin-like peptide induces bacteriocin synthesis in L. plantarum C11. Mol Microbiol 18, 631-639.

Eijsink, V. G. H., Brurberg, M. B., Middelhoven, P. H. \& Nes, I. F. (1996). Induction of bacteriocin production in Lactobacillus sake by a secreted peptide. J Bacteriol 178, 2232-2237.

Franz, C. M. A. P., Schillinger, U. \& Holzapfel, W. H. (1996). Production and characterization of enterocin 900, a bacteriocin produced by Enterococcus faecium BFE 900 from black olives. Int $J$ Food Microbiol 29, 255-270.
Franz, C. M. A. P., van Belkum, M. J., Worobo, R. W., Vederas, J. C. \& Stiles, M. E. (2000). Characterization of the genetic locus responsible for production and immunity of carnobacteriocin A: the immunity gene confers cross-protection to enterocin B. Microbiology 146, 621-631.

Franz, C. M. A. P., van Belkum, M. J., Holzapfel, W. H., Abriouel, H. \& Gálvez, A. (2007). Diversity of enterococcal bacteriocins and their grouping in a new classification scheme. FEMS Microbiol Rev 31, 293-310.

Gursky, L. J., Martin, N. I., Derksen, D. J., van Belkum, M. J., Kaur, K., Vederas, J. C., Stiles, M. E. \& McMullen, L. M. (2006). Production of piscicolin 126 by Carnobacterium maltaromaticum UAL26 is controlled by temperature and induction peptide concentration. Arch Microbiol 186, 317-325.

Håvarstein, L. S., Diep, D. B. \& Nes, I. F. (1995). A family of bacteriocin $\mathrm{ABC}$ transporters carry out proteolytic processing of their substrates concomitant with export. Mol Microbiol 16, 229-240.

Johnsborg, O., Diep, D. B. \& Nes, I. F. (2003). Structural analysis of the peptide pheromone receptor $\mathrm{PlnB}$, a histidine protein kinase from Lactobacillus plantarum. J Bacteriol 185, 6913-6920.

Kaiser, E., Colescott, R. L., Bossinger, C. D. \& Cook, P. I. (1970). Color test for detection of free terminal amino groups in the solidphase synthesis of peptides. Anal Biochem 34, 595-598.

Keller, L. \& Surette, M. G. (2006). Communication in bacteria: an ecological and evolutionary perspective. Nat Rev Microbiol 4, 249-258.

Klaenhammer, T. R. (1993). Genetics of bacteriocins produced by lactic acid bacteria. FEMS Microbiol Rev 12, 39-86.

Kleerebezem, M. \& Quadri, L. E. (2001). Peptide pheromonedependent regulation of antimicrobial peptide production in Grampositive bacteria: a case of multicellular behavior. Peptides 22, 1579-1596.

Kleerebezem, M., Quadri, L. E. N., Kuipers, O. P. \& de Vos, W. M. (1997). Quorum sensing by peptide pheromones and two-component signal-transduction systems in Gram-positive bacteria. Mol Microbiol 24, 895-904.

Kleerebezem, M., Kuipers, O. P., de Vos, W. M., Stiles, M. E. \& Quadri, L. E. N. (2001). A two-component signal-transduction cascade in Carnobacterium piscicola LV17B: two signaling peptides and one sensor-transmitter. Peptides 22, 1597-1601.

Kristiansen, P. E., Fimland, G., Mantzilas, D. \& Nissen-Meyer, J. (2005). Structure and mode of action of the membrane-permeabilizing antimicrobial peptide pheromone plantaricin A. J Biol Chem 280, 22945-22950.

Morrow, J. A., Segall, M. L., Lund-Katz, S., Phillips, M. C., Knapp, M., Rupp, B. \& Weigraber, K. H. (2000). Differences in stability among the human apolipoprotein $\mathrm{E}$ isoforms determined by the amino-terminal domain. Biochemistry 39, 11657-11666.

Mutter, M., Nefzi, A., Sato, T., Sun, X., Wahl, F. \& Wöhr, T. (1995). Pseudo-prolines (psi Pro) for accessing 'inaccessible' peptides. Pept Res 8, 145-153.

Nes, I. F., Diep, D. B., Håvarstein, L. S., Brurberg, M. B., Eijsink, V. \& Holo, H. (1996). Biosynthesis of bacteriocins in lactic acid bacteria. Antonie Van Leeuwenhoek 70, 113-128.

Nilsen, T., Nes, I. F. \& Holo, H. (1998). An exported inducer peptide regulates bacteriocin production in Enterococcus faecium CTC492. J Bacteriol 180, 1848-1854.

Reading, N. C. \& Sperandio, V. (2006). Quorum sensing: the many languages of bacteria. FEMS Microbiol Lett 254, 1-11.

Rohde, B. H. \& Quadri, L. E. N. (2006). Functional characterization of a three-component regulatory system involved in quorum sensing-based 
regulation of peptide antibiotic production in Carnobacterium maltaromaticum. BMC Microbiol 6, 93.

Saucier, L., Poon, A. \& Stiles, M. E. (1995). Induction of bacteriocin in Carnobacterium piscicola LV 17. J Appl Bacteriol 78, 684-690.

van Belkum, M. J. \& Stiles, M. E. (2000). Nonlantibiotic antibacterial peptides from lactic acid bacteria. Nat Prod Rep 17, 323-335.

van Belkum, M. J., Worobo, R. W. \& Stiles, M. E. (1997). Doubleglycine-type leader peptides direct secretion of bacteriocins by $\mathrm{ABC}$ transporters: colicin V secretion in Lactococcus lactis. Mol Microbiol 23, 1293-1301.
Vaughan, A., Eijsink, V. G. H. \& van Sinderen, D. (2003). Functional characterization of a composite bacteriocin locus from malt isolate Lactobacillus sakei 5. Appl Environ Microbiol 69, 7194-7203.

Worobo, R. W., Henkel, T., Sailer, M., Roy, K. L., Vederas, J. C. \& Stiles, M. E. (1994). Characteristics and genetic determinant of a hydrophobic peptide bacteriocin, carnobacteriocin A, produced by Carnobacterium piscicola LV17A. Microbiology 140, 517-526.

Edited by: M. Kleerebezem 